\title{
Complications of Hip Arthroplasty
}

\section{Editorial Comment}

\author{
R. Michael Meneghini MD
}

Published online: 22 June 2010

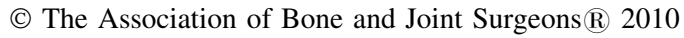

Total hip arthroplasty has become one of the most predictable and reproducible procedures in medicine; however, while uncommon, complications continue to occur and remain potentially devastating to both patient and surgeon alike. Therefore, as we strive to improve an already reliable surgical procedure, it has become increasingly important that these improvements target the prevention and treatment of complications in order to achieve better short- and long-term outcomes. Furthermore, as the economics of rising costs continue to drive healthcare reform to optimize outcomes while promoting efficiency, it is imperative that arthroplasty surgeons focus on minimizing complications to enhance the value of hip arthroplasty within our society and the healthcare market. Therefore, we believe the content of this symposium timely and relevant to any orthopaedic surgeon who performs hip arthroplasty today.

Recently, modern surgical techniques and implant biomaterials have been developed with claims of improving outcomes, yet conflicting reports of increased and occasionally unique complications have emerged. This has created controversy and tempered enthusiasm for these new technologies, as well as advising caution for rapid acceptance and utilization. As physicians and as a profession, we are obligated to critically evaluate new techniques and technologies with strict scientific rigor and make informed treatment decisions in an evidence-based fashion. Some of the current and most relevant issues that arthroplasty surgeons are facing include hip resurfacing

\section{R. Michael Meneghini ( $\square)$}

Department of Orthopaedic Surgery, University of Connecticut

Health Center, 263 Farmington Avenue, MARB 4th Floor,

Farmington, CT 06034, USA

e-mail: rm_meneghini@yahoo.com arthroplasty, alternative bearing surfaces and minimal incision surgical techniques. Of particular interest are the unique complications of metal-on-metal bearings and hip resurfacing that have recently followed a period of increased usage by surgeons. Metal-on-metal bearings have the benefit of decreased wear and large-diameter heads that provide added stability, yet complications related to metal hypersensitivity and wear have tempered enthusiasm for this bearing surface and have called into question whether the proposed benefits of this new technology will truly decrease complications and improve patient outcomes. We are fortunate to have all of these modern issues addressed critically by various articles in this symposium.

I would like to personally thank all of the contributing authors for their excellent research presented in this symposium. The topics are timely and relevant and the quality of the work is exceptional. I would also like to thank the reviewers who contributed their time and expertise to critically review all the manuscripts and uphold strict

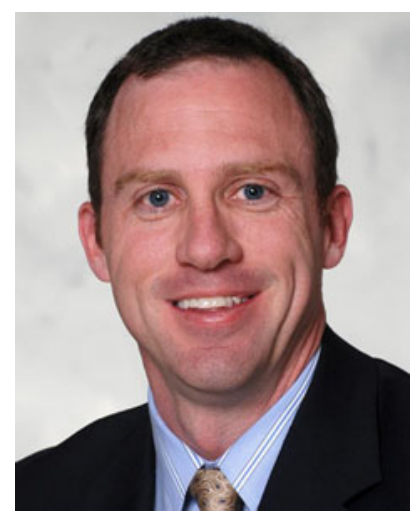

Fig. 1 R. Michael Meneghini, MD is shown. 
scientific rigor. Finally, I would like to extend my sincere appreciation and personal gratitude to Paul Lotke and Dick Brand. Their tireless efforts and talents brought this symposium from concept to reality and it would not have been possible without them. I am hopeful the readership of CORR will enjoy and benefit from the outstanding works presented in this symposium and appreciate the critical scientific review involved. It is our goal that the information presented here allows the orthopaedic surgeon to make more informed and evidence-based decisions regarding the prevention and treatment of hip arthroplasty complications, improving safety and outcomes for patients. 